\title{
WHICH OPERATORS ARE THE SELF-COMMUTATORS OF COMPACT OPERATORS?
}

\author{
PENG FAN ${ }^{1}$ AND CHE-KAO FONG
}

\begin{abstract}
It is shown that, for a hermitian operator $T$ on a Hilbert space $H$, the following three statements are equivalent: (i) $T=A^{*} A-A A^{*}$ for some compact operator $A$, (ii) there is an orthonormal basis $\left\{b_{j}\right\}$ such that $\left\langle T b_{j}, b_{j}\right\rangle=0$ for all $j$, and (iii) $\operatorname{tr} T^{+}=\operatorname{tr} T^{-}$(possibly infinite) where $T^{+}=(|T|+T) / 2$ and $T^{-}=$ $(|T|-T) / 2$.
\end{abstract}

For a bounded operator $A$ on a (separable, infinite dimensional) Hilbert space $H$, the self-commutator of $A$, denoted by $\left[A^{*}, A\right]$, is defined to be $A^{*} A-A A^{*}$. The question concerning which operators are self-commutators was answered by Radjavi ([6]; also see [1]) as follows: a hermitian operator is a self-commutator (of some operator) if and only if its essential numerical range contains zero. The purpose of the present paper is to supply an answer to the compact counterpart of the above question, a problem posed by Pearcy [4, Problem III], as follows:

THEOREM 1. Let $T$ be a hermitian compact operator. Then the following three statements are equivalent:

(i) $T=\left[A^{*}, A\right]$ for some compact operator $A$.

(ii) There exists an orthonormal basis $\left\{b_{j}\right\}$ such that $\left\langle T b_{j}, b_{j}\right\rangle=0$ for all $j$.

(iii) $\operatorname{tr} T^{+}=\operatorname{tr} T^{-}$where $T^{+}=(|T|+T) / 2$ is the positive part of $T$ and $T^{-}=(|T|-T) / 2$ is the negative part of $T$.

(Here $|T|$ is the positive square root of $T^{2}$. For the definition and basic properties of $\operatorname{tr} P$ for a positive operator $P$, see [3].)

For the proof of this theorem, we need a technical lemma.

LEMMA 2. Let $\left\{p_{j}\right\}$ be a sequence (possibly finite) of nonnegative numbers and $\left\{-q_{j}\right\}$ be an infinite sequence of negative numbers such that $\Sigma p_{j}=\Sigma q_{j}< \pm \infty, q_{j} \rightarrow$ 0 as $j \rightarrow \infty$ and $p_{j} \rightarrow 0$ in case $\left\{p_{j}\right\}$ is an infinite sequence. Then these two sequences can be arranged into one sequence $\left\{r_{j}\right\}$ such that

(i) $s_{n}=r_{1}+r_{2}+\cdots+r_{n} \geqslant 0$ for all $n$, and

(ii) $s_{n} \rightarrow 0$ as $n \rightarrow \infty$.

The proof of the above lemma is elementary and hence omitted.

Received by the editors May 21, 1979 and, in revised form, August 24, 1979.

AMS (MOS) subject classifications (1970). Primary 47B47; Secondary 47B05.

Key words and phrases. Self-commutator, compact operator, trace.

${ }^{1}$ The first author presents a part of his dissertation written under the direction of Professor J. G. Stampfli. 
Proof of Theorem 1. We will show that (i) implies (ii), (ii) implies (iii) and (iii) implies (i).

(i) implies (ii). Suppose that $T=\left[A^{*}, A\right]$ where $A$ is a compact operator. Write $A=B+i C$ where $B, C$ are hermitian compact operators. Then $T=2 i[B, C]$. Let $\left\{b_{j}\right\}$ be an orthonormal basis consisting of eigenvectors of $B$, say $B b_{j}=\lambda_{j} b_{j}$, where $\lambda_{j}$ is a real number. We have

$$
\begin{aligned}
\left\langle T b_{j}, b_{j}\right\rangle & =2 i\left\langle(B C-C B) b_{j}, b_{j}\right\rangle \\
& =2 i\left\langle C b_{j}, B b_{j}\right\rangle-2 i\left\langle C B b_{j}, b_{j}\right\rangle \\
& =2 i\left\langle C b_{j}, \lambda_{j} b_{j}\right\rangle-2 i\left\langle C\left(\lambda_{j} b_{j}\right), b_{j}\right\rangle=0 .
\end{aligned}
$$

(ii) implies (iii). Suppose that $\left\{b_{j}\right\}$ is an orthonormal basis such that $\left\langle T b_{j}, b_{j}\right\rangle=$ 0 for all $j$. Since $T^{+}=T+T^{-}$, we have

$$
\begin{aligned}
\operatorname{tr} T^{+} & =\Sigma\left\langle T^{+} b_{j}, b_{j}\right\rangle=\Sigma\left(\left\langle T b_{j}, b_{j}\right\rangle+\left\langle T^{-} b_{j}, b_{j}\right\rangle\right) \\
& =\Sigma\left\langle T^{-} b_{j}, b_{j}\right\rangle=\operatorname{tr} T^{-} .
\end{aligned}
$$

(iii) implies (i). Assume that $\operatorname{tr} T^{+}=\operatorname{tr} T^{-}$. Since $T$ is compact and hermitian, it is unitarily equivalent to a diagonal operator, say

$$
T_{0}=\operatorname{diag}\left\{d_{1}, d_{2}, d_{3}, \ldots\right\} .
$$

We may assume that there are infinitely many $j$ with $d_{j}<0$. (Otherwise, either $-T_{0}$ has infinitely many negative eigenvalues or $T_{0}$ is a finite rank operator, both cases can be handled by modifying the following argument.) From $\operatorname{tr} T^{+}=\operatorname{tr} T^{-}$, we obtain

$$
\Sigma\left\{d_{j}: d_{j} \geqslant 0\right\}=\Sigma\left\{d_{j}: d_{j}<0\right\} .
$$

By Lemma 1, we can rearrange the sequence $\left\{d_{j}\right\}$ in such a way that $s_{n}=d_{1}+d_{2}$ $+\cdots+d_{n} \geqslant 0$ for all $n$ and $s_{n} \rightarrow 0$ as $n \rightarrow \infty$. Let $A_{0}$ be the weighted shift on $l^{2}$ defined by

$$
A_{0}\left(x_{1}, x_{2}, x_{3}, \ldots\right)=\left(0, \sqrt{s_{1}} x_{1}, \sqrt{s_{2}} x_{2}, \ldots\right) .
$$

Then $A_{0}$ is compact and $T_{0}=\left[A_{0}^{*}, A_{0}\right]$. Now it is clear that $T$ is the self-commutator of a compact operator. The proof is complete.

COROllary 3. Every compact hermitian operator is a sum of two self-commutators of compact operators.

Proof. Let $T$ be a compact hermitian operator. By considering $-T$ if necessary, we may assume that $T$ is an operator of the form

$$
T=\operatorname{diag}\left\{p_{1}, p_{2}, p_{3}, \ldots\right\} \oplus A
$$

with $p_{j} \geqslant 0$ and $A \leqslant 0$. (We do not exclude the possibility that $A$ is absent.) Let

$$
\begin{aligned}
& T_{1}=\operatorname{diag}\left\{p_{1}+1,-\frac{1}{2}, p_{3}+\frac{1}{3},-\frac{1}{4}, p_{5}+\frac{1}{5}, \ldots\right\} \oplus A, \\
& T_{2}=\operatorname{diag}\left\{-1, p_{2}+\frac{1}{2},-\frac{1}{3}, p_{4}+\frac{1}{4},-\frac{1}{5}, \ldots\right\} \oplus 0 .
\end{aligned}
$$


Then $T=T_{1}+T_{2}$. Since $\operatorname{tr} T_{1}^{+}=\operatorname{tr} T_{1}^{-}=\operatorname{tr} T_{2}^{+}=\operatorname{tr} T_{2}^{-}=+\infty$, by Theorem 1 , both $T_{1}$ and $T_{2}$ are self-commutators. The proof is complete.

REMARKS. (1) It follows from the above corollary that every compact operator can be expressed as a sum of at most four commutators of compact operators. This slightly improves the following result in [5]: every compact operator is a sum of finitely many commutators of compact operators.

(2) It follows from Theorem 1 that if a positive operator is the self-commutator of a compact operator, then it must be zero. This gives a fresh look of an early result for hyponormal operators, namely, compact hyponormal operators are normal. (See, e.g., [7].)

(3) From the proof of Theorem 1, we see that each of the following conditions are equivalent to each of those in Theorem 1:

(iv) $T=\left[Q^{*}, Q\right]$ for some compact quasi-nilpotent weighted shift $Q$.

(v) $T=\left[S^{*}, S\right]$ for some operator $S$ with its real part $\left(S+S^{*}\right) / 2$ compact.

(4) It follows from [2] and [6] that real parts of quasi-nilpotent operators and self-commutators have the same characterizaton. However, by a result in [2], every compact hermitian operator is the real part of a compact quasi-nilpotent operator, while, in view of Theorem 1 , a compact hermitian operator is not necessarily the self-commutator of a compact operator.

(5) Note that the set of all self-commutators is closed under the norm-topology, while the set of all self-commutators of compact operators is not so.

The authors would like to thank the referee for pointing out that, pertinent to Remark (1), every compact operator is a sum of at most three commutators of compact operators. The argument for proving this fact is reproduced here as follows. For a compact operator $T$, write $T=H+i K$, where $H$ and $K$ are compact hermitian operators. By Corollary $3, H=\left[A^{*}, A\right]+\left[B^{*}, B\right]$ for some compact operators $A, B$. Observe that $H=\operatorname{Re} S$, where $S=\left[A^{*}+i B, A+i B^{*}\right]$. Hence $T=S+i(K-\operatorname{Im} S)$ and, by Corollary 3 again, $K-\operatorname{Im} S$ is the sum of two commutators of compacts.

\section{REFERENCES}

1. A. Brown, P. R. Halmos and C. Pearcy, Commutators of operators on Hilbert spaces, Canad. J. Math. 17 (1965), 695-708.

2. P. Fillmore, C. K. Fong and A. Sourour, Real parts of quasi-nilpotent operators, Proc. Edinburgh Math. Soc. (to appear).

3. I. C. Gohberg and M. G. Krein, Introduction to the theory of linear non-self-adjoint operators, Transl. Math. Monographs, vol. 18, Amer. Math. Soc., Providence, R. I., 1969.

4. C. Pearcy, Some unsolved problems in operator theory, preprint, 1972.

5. C. Pearcy and D. Topping, On commutators in ideal of compact operators, Michigan Math. J. 18 (1971), 247-252.

6. H. Radjavi, Structure of $A^{*} A-A A^{*}$, J. Math. Mech. 16 (1966), 19-26.

7. J. G. Stampfli, Hyponormal operators, Pacific J. Math. 12 (1962), 1453-1458.

Department of MATHEMatics, Indiana UNIVERSITY, BloOMngGTON, INDIANA 47401

Department of Mathematics and Statistics, University of Guelph, Guelph, Nig 2 W1 Ontario, Canada 